\title{
Four whole-istic aspects of schistosome granuloma biology: fractal arrangement, internal regulation, autopoietic component and closure
}

\author{
HL Lenzi/+ , W de S Romanha, RM Zorzenon dos Santos*, A Rosas**, EM Mota, \\ PPA Manso, LFG Caputo, M Pelajo-Machado
}

Departamento de Patologia, Instituto Oswaldo Cruz-Fiocruz, Av. Brasil 4365, 21040-900 Rio de Janeiro, RJ, Brasil

*Departamento de Física, Universidade Federal de Pernambuco, Recife, PE, Brasil **Departamento de Física, Universidade Federal da Paraíba, João Pessoa, PB, Brasil

This paper centers on some whole-istic organizational and functional aspects of hepatic Schistosoma mansoni granuloma, which is an extremely complex system. First, it structurally develops a collagenic topology, originated bidirectionally from an inward and outward assembly of growth units. Inward growth appears to be originated from myofibroblasts derived from small portal vessel around intravascular entrapped eggs, while outward growth arises from hepatic stellate cells. The auto-assembly of the growth units defines the three-dimensional scaffold of the schistosome granulomas. The granuloma surface irregularity and its border presented fractal dimension equal to 1.58. Second, it is internally regulated by intricate networks of immuneneuroendocrine stimuli orchestrated by leptin and leptin receptors, substance $P$ and Vasoactive intestinal peptide. Third, it can reach the population of \pm 40,000 cells and presents an autopoietic component evidenced by internal proliferation (Ki- $\left.67^{+} \mathrm{cells}\right)$, and by expression of $\mathrm{c}-\mathrm{Kit}^{+} \mathrm{cell}$, leptin and leptin receptor (Ob-R), granulocyte-colony stimulating factor (G-CSF-R), and erythropoietin (Epo-R) receptors. Fourth, the granulomas cells are intimately connected by pan-cadherins, occludin and connexin-43, building a state of closing (granuloma closure). In conclusion, the granuloma is characterized by transitory stages in such a way that its organized structure emerges as a global property which is greater than the sum of actions of its individual cells and extracellular matrix components.

Key words: Schistosoma mansoni - granuloma - fractal - leptin - ocludin - connexin-43

Conservative approaches in science usually try to explain most of the dynamical systems as the result of the interplay between its elementary units. In general these unities are investigated independently of each other without taking into account its interactions and cooperative phenomena. More contemporary approaches try to understand the system as a whole taking into account its organizational structure, the mechanisms underlying its dynamical behavior, the information exchanging between unities, and so on (Chong \& Bryan Ray 2002). Biological systems for a long time have been studied and described without taking into account the wholeness of the system. As a consequence, their emergent properties that may result from collective and cooperative phenomena among its elements or unities in general, could not be reproduced properly in these attempts. New approaches that take into account the main interaction among the different elements (cells, proteins, etc.) that compose biological system has been shown appropriate to describe the emergent properties exhibited by these systems, therefore allowing the comparison between experimental and mathematical re-

Financial support: CNPq, Fiocruz

${ }^{+}$Corresponding author: henrique.lenzi@gmail.com

Received 25 May 2006

Accepted 26 June 2006 sults or the understanding of the mechanisms underlying some pattern observed.

Schistosome granuloma, as those caused by Mycobacterium tuberculosis and other granuloma types, is a complex spatial-temporal (structure) system formed by components that interact in an intricate way, so it can not be described by simplistic models. The number of elements (cells and signaling molecules) that interact to build such structure are so many that its global behavior can only be understood as an emerging consequence derived from the integral sum of all range of the cooperative phenomena (Lenzi \& Romanha 2003, Lenzi et al. 2004).

The granuloma presents the following life cycle: (i) encapsulating and focal histolysis: comprehends a very quick process, when many cells such as monocytes and eosinophils, with few lymphocytes, form a cellular cushion around the egg. These cells intensively diffuse to the perivascular tissue (outward cellular wave), and together with cells that are chemo-attracted from the sinusoids (inward cellular wave), define a loose and disarranged cluster of many cells (mainly eosinophils and monocytes) that take over the damaged local tissue; (ii) fiber or extracellular matrix production: characterizes the early stage of exudative-productive phase; (iii) orientation and compacting: are expressed by more compact arrangement of collagen fibrous mesh; (iv) involution and disintegration: begin after the spontaneous death of the miracidia, arousing out of collagen degradation, subsiding of the inflammatory reaction, until its final disappearance (Lenzi et al. 1998, Lenzi \& Romanha 2003). 
The formation of schistosome granuloma can be divided into two major stages: pre-granulomatous (initial reactive and exudative phases) and granulomatous (exudative-productive, productive, advanced or fibrotic-productive, and involutional phases) (Lenzi et al. 1998). The former stage is characterized by disorganized aggregation of cells and the latter one by organization of them in the exudative-productive and productive phases, followed by the involutional process.

As any other biological system, the understanding of the granuloma formation will be based on adequate technologies to obtain the full description of the different stages and methodologies to assemble all the information (data) obtained and highlight the essential mechanisms/ features for the integration of the parts and the stability of the whole. Interdisciplinary approaches can contribute to the development of new technologies (and tools) as well as to the methodologies that may improve the data analysis.

This paper focuses on the study of some whole-istic, organizational and functional, aspects of Schistosoma mansoni granuloma, which is an extremely complex system (Lenzi \& Romanha 2003, Lenzi et al. 2004), tending to circumvent the egg products.

\section{MATERIALS AND METHODS}

Ninety-nine Swiss Webster mice were infected when they were five days old by percutaneous exposure to 70 cercariae of the Belo Horizonte isolate of S. mansoni, and were sequentially submitted to euthanasia by ether inhalation in accordance with the animal ethical practice, at $45,50,60,65,70,80,85,95,100,120$, and 160 days after infection. Liver sections of six animals/point of infection were divided; part was fixed in formalin-Millonig (Carson et al. 1973) and embedded in paraffin and part was frozen in liquid nitrogen to immunostainings. Sections $(5 \mu \mathrm{m})$ were stained with Hematoxilin-Eosin (HE); Lennert's Giemsa; Sudan III, PAS; PAS-Alcian Blue pH 2.5 e 1.0; Perls; Picrosirius conterstained with HE or light green; Feulgen with Merck kit; Jone's hexamine-silver stain; Sirius red $\mathrm{pH}$ 10,2 (for eosinophils); Phosphomolibdic acidPicrosirius Red (PMA-PSR) (for interstitial collagens) (Dolber \& Spach 1993), and Direct Blue with or without oxone oxidation [for elastic fibers (Caputo et al., not published)]. The slides stained with Feulgen, Sirius Red $\mathrm{pH}$ 10.2, Jone's hexamine-silver stain, PMA-PSR, and Direct Blue were analyzed by confocal laser scanning microscopy (LSM 510 - META, Zeiss). The fractal study was done in sections stained with Feulgen, Jone's hexaminesilver stain and PMA-PSR.

Indirect immnunofluorescence was done on cryostat liver sections $(5-6 \mu \mathrm{m})$ applying, at $37^{\circ} \mathrm{C}$ for $1 \mathrm{~h}$, primary antibodies against collagen I and III, fibronectin, tenascin, vitronectin, Ki-67 (Dako, CA, US), pan-cadherin (SigmaAldrich Co, US - C3678), occludin, connexin-43, leptin, leptin-receptor (Ob-R), granulocyte colony stimulating factor-receptor (G-CSF-R) and erythropoietin-receptor (Epo-R) (Santa Cruz Biotechnology Inc., CA, US). Depending on the primary antibody, secondary anti-rabbit or antimouse antibody coupled to AlexaFluor 488 (Molecular Probe, US) was used, counterstained with 1/10,000 Evans
Blue. Sections were coverslipped in buffered glycerin with p-phenylenediamine and the slides were analyzed by confocal microscopy on a Zeiss LSM 510-META.

The liver of other three animals/point infection were used to obtain isolated granulomas, according to Brenner and Pellegrino (1956) procedure. From each liver, 120 complete intact granulomas were individually isolated by suction under stereomicroscope, and cells were dispersed with collagenase I ( $1 \mathrm{mg} / \mathrm{ml}$ in $3 \mathrm{ml}$ for 120 granulomas) at $37^{\circ} \mathrm{C}$ for $60 \mathrm{~min}$, with vigorous homogenization each 15 min. The total cells were counted in Neubauer chamber, after stain with Türk solution and negrosin stain for livedead ratio. The total number of viable cells was divided by 120 , to figure out the total population of individual granuloma at each point of infection.

The morphologic aspects of fractals were verified in PAMS (PAS-metanamine silver) and PMA-PSR slides, by virtual tomography and three-dimensional modeling applying software from laser scanning confocal microscopy (LSM 510 - META, Zeiss). The fractal dimension of the granuloma external border was estimated from Feulgen stained slides, in liver specimens from 45, 50, 60, and 70 days after infection, using Hurst exponent.

\section{RESULTS}

Structural characteristics of the extracellular matrix - Hepatic schistosome granulomas showed, mainly in the exudative-productive stage, different patterns of production and deposition of extracellular matrix components (interstitial collagens I and III, tenascin, vitronectin, proteoglycans, hyaluran, and elastic fibers). Fibronectin, vitronectin, tenascin, and interstitial collagens predominated in the middle or paracentral zone of the granulomas (Fig. 1). In mature exudative-productive granulomas, the fibers in the peripheral zone showed a looser arrangement, while in the middle zone they were more compact, but always forming a storiform mesh, with fiber irradiation centers (Figs 2-4). In the exudative-productive phase, the fibrous mesh of the middle zone became more compact, parallel and concentric, acquiring a circumferential arrangement (Fig. 5). The elastic fibers predominated more in the external or peripheral zone and in the interface between peripheral and middle zones, defining a border where eosinophils were centrally placed (Fig. 6) and pigmented macrophages peripherally located (Fig. 7). The three-dimensional analysis of tissue architecture of the granuloma during its development, as observed by confocal laser scanning microscopy, showed that the granulomas develop a variable and irregular collagenic topology bidirectionally oriented. Part of the interstitial collagen appears to be originated from myofibroblasts derived from small portal vessel around intravascular entrapped eggs. In another way, there is also an outward collagenic growth, usually around $100 \mu \mathrm{m}$ far away from the eggs, which arises from hepatic stellate cells [lipocytes, Ito cells, perisinusoidal cells (HSCs)] (Figs 8-10). Each collagenic growth unit acts as a fibrotic center from which irradiate four irregular and sinuous projections that eventually branch, generating, in general, three or four interactions or "axonal" extensions $(\mathrm{n}=3-4)$ (Figs 8-11). Each unit is originated from multiple cells, and the "axonal" extensions 


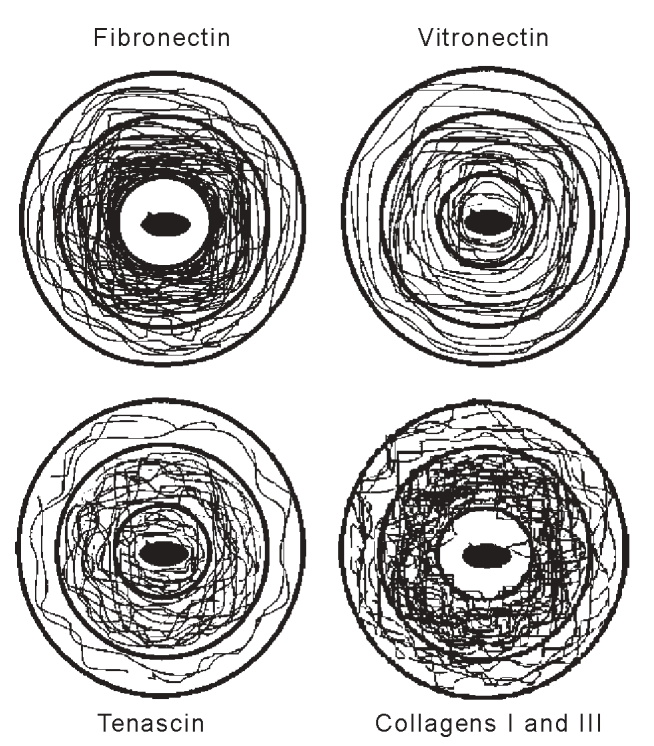

Fig. 1: scheme showing the distribution of different extracellular components in the three zones (external or peripheral, medial or intermediate and central or periovular) of schistosome exudativeproductive granuloma.

gradually interconnect with extensions from adjacent units (Figs 11-13). The structure developed in the interface between granulomas and hepatocytes, gradually, starts to define a peripheral closure of the granulomas (Figs 1415). The auto-assembly of these connections defines the three-dimensional scaffold of the granulomas, which acquires structural characteristics like-tensegrity (Figs 1619).

Expression of adipokines and neuropeptides by schistosome granulomas - Exudative-productive granulomas highly expressed leptin and leptin receptor. The leptin was located in the three granuloma zones, while the leptinreceptors predominated in the external one (Figs 20-22). Otherwise, substance $P$ and vasoactive intestinal peptide (VIP) were only localized in the central or peri-ovular zone (Figs 23, 24).

Evidence of internal cellular production by granulomas and expression of hematopoietin-receptors - Hepatic granulomas reached the population of more than 40,000 cells (the sum of migrated and internal cells) in the acute phase (45 days after infection), followed by a plateau around 30,000 cells (from 50 to 70 days after infection), decreasing gradually until 95 and 100 days after infection) (Fig. 36). Eosinopoietic foci are often and easily seen in the peripheral or external zone (Figs 25, 26) and evidence of cellular proliferation was mainly observed in the peripheral and central zones by Ki-67 marker (Figs 27, 28). Expression of c-Kit (Fig. 29), receptors of granulocyte colony stimulating factor (G-CSF-R) (Fig. 30) and erythropoietin (Epo-R) (Fig. 31) predominated in the peripheral and central zones.

Granuloma closure - The granuloma cells were intimately connected by pan-cadherins (Fig. 32), occludin (Fig. 33), and connexin-43 (Fig. 34), building a state of closing (granuloma closure). Eosinophils, sometimes, degranulated and die on the surface of egg shells, forming a crystallized protein barrier, defining aspect corresponding to Splendore-Hoeppli phenomenon (Fig. 35).

\section{DISCUSSION}

This paper focused on four characteristic aspects of the schistosomal granuloma as a whole: (i) it shows, structurally, different patterns of production and deposition of extracellular matrix components developing a variable and irregular collagenic topology, originated bidirectionally from an inward and an outward assembly of growth units; (ii) it presents internal production of neuropeptides; (iii) it can reach the population of more than 40.000 cells in the acute phase and presents an autopoietic component evidenced by internal production ( $\mathrm{Ki}-67^{+}$cells), expression of immature cells $\left(\mathrm{c}-\mathrm{Kit}^{+}\right)$, hematopoietic growth factors and their respective receptors (leptin and leptin receptors), granulocyte colony stimulating factor receptor (GCSF-R) and erythropoietin receptor (Epo-R); (iv) its cells are intimately connected by cadherins, tight junctions (occludin) and gap junctions (connexin-43), building a state of closing (granuloma closure).

Inward collagenic growth appears to be originated from myofibroblasts derived from small portal vessel around intravascular entrapped eggs, while the outward growth, usually around $100 \mu \mathrm{m}$ away from the eggs, arise from hepatic stellate cells (HSCs) (lipocytes, Ito cells, perisinusoidal cells). Each growth unit acts as a fibrotic center as was previously described (Lenzi et al. 1999). However, it is not formed by a single cell as was thought before, but it is originated in the interface between three or more cells, depending on the framework of the cellular arrangement, which determines the curvature or angles between collagen fibers (Figs 12,13). The application of fractal analysis to evaluate the fibrosis development or fibrogenesis and the fibrosis intensity will probably bring much more information than the traditional methods used to measure or evaluate irregular biologic structures which cannot be correctly quantified by Euclidean-based metric methodologies (Dioguardi et al. 2003). In classical Euclidean geometry, objects have integer dimensions: a point $=$ 0 (zero dimension), a line $=1$ (one dimension: length), a plane $=2$ (two dimensions: length and width), a volume $=$ 3 (three dimensions: length, width and depth). Euclidean geometry is useful for describing manmade objects or ideal shapes, but natural shapes are much more complex (Landini 1996). For instance, the branching structure of a vascular tree or the irregular membrane of a lymphocyte can not be associated to any classical shape. Moal et al. (2003) have shown that fractal analysis is very suitable for analyzing liver fibrosis, having excellent reproducibility. It is the only quantitative morphometric method that can discriminate among the models of fibrosis and be sensitive to detect pharmacologically induced changes in liver fibrosis. Study of the fractal properties of the liver (or of the granuloma) is likely to reveal more about its structure and the pathogenesis of liver diseases (Dioguardi et al. 1999). There are two types of fractals based on the strict meaning of similarity. One group is composed of objects whose smaller scales replicate exactly the larger ones ("exact-self-simi- 

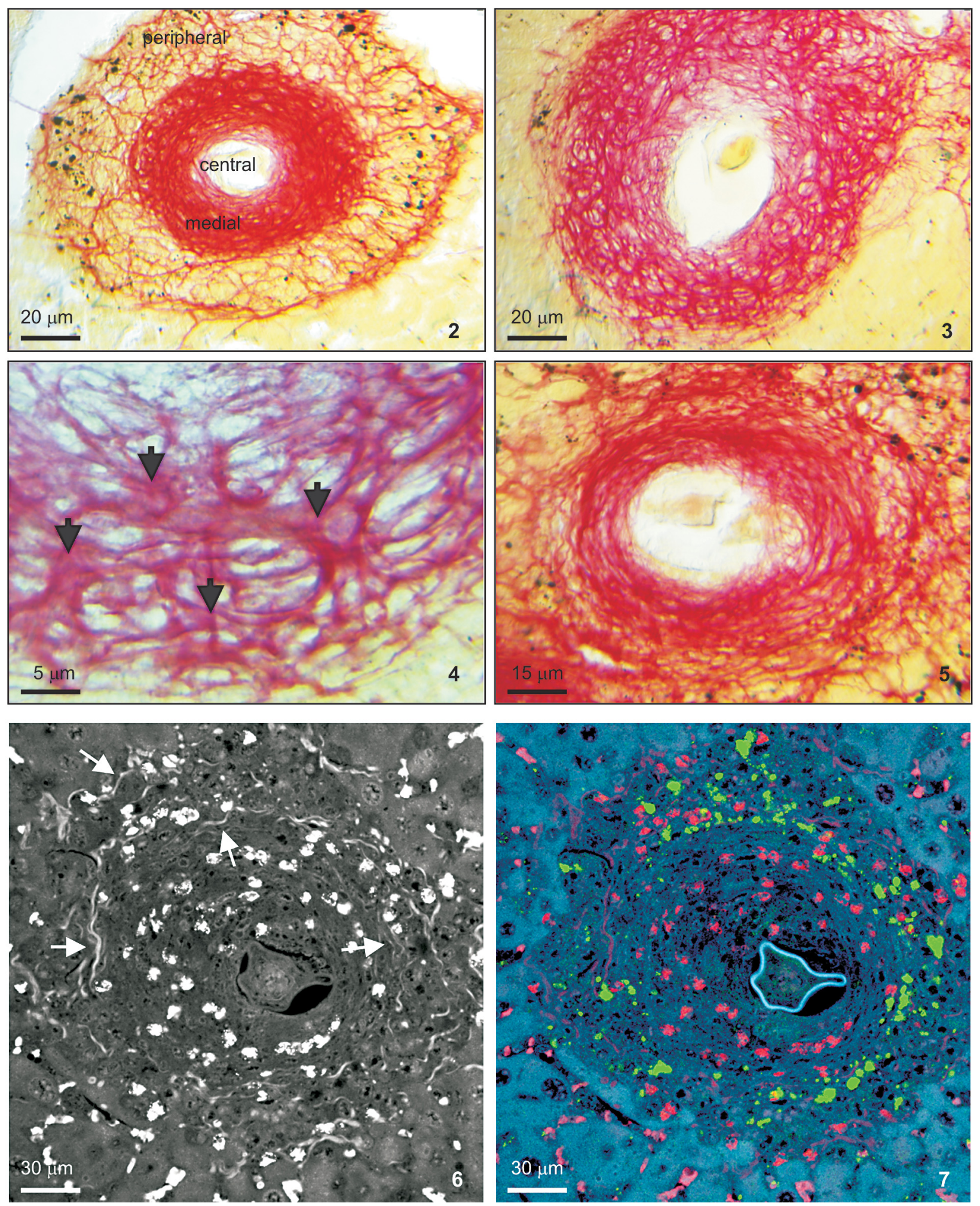

Fig. 2: exudative-productive granuloma showing the collagen arrangement in the very well defined three zones. The external or peripheral zone is expanded and forms a loose stroma adequate to intragranulomatous hematopoietic metaplasia. The medial zone presents a denser collagen mesh, which is better seen in Figs 3 and 4: this mesh is defined by the confluence among different fiber radiation centers (arrows in Fig. 4). The central zone has few and delicate fibers, been devoid of collagen in the periovular subregion. Fig. 5: productive stage with a compact concentric arrangement in the medial zone (Picrosirius). Fig. 6: elastic fiber, labeled by Direct Blue, located mainly in the interface between the external zone with the hepatic parenchyma and the medial zone (arrows). Fig. 7: elastic fibers define different topographic areas inside the granuloma, limiting the internal ingression of pigmented macrophages (green cells), without interfering with internal eosinophil migration (red cells). 
lar"). The second group is made up of "statistically selfsimilar" or "random" fractals, which differs from the former by the fact that the reproduced detail in different scales is not an exact copy of each other but statistically the same (Landini 1996). The granuloma seems to correspond to fractals of the second type ("random" fractals). In the granuloma fibrosis, each growth unit operates as a fiber radiation center in the fibrotic diffusion process. Preliminary results, based on the evaluation of Hurst exponent, indicated that the fractal dimension of the border of schistosomal granuloma is $\mathrm{D}=1.58$. The fractal dimension corresponds to an index of the space-filling properties of an object in its corresponding Euclidean (topological) dimension so that the closer the dimension is to the topological dimension in which the object is embedded, the greater its space-filling properties (Cross 1997). When the object completely fills the space in which it is embedded, the fractal dimension reaches the same value of the topological dimension. The bulk fractal dimension, which may be different in each granuloma stage, and the multifractal spectrum, which may give information about the geometrical aspects of the distinct zones, will be subject of future studies. The final architectural arrangement of the interstitial collagens in the exudative-productive granuloma, defines clearly three zones (Lenzi et al. 1998) and acquires a general aspect very similar to a stable symmetric tensegrity. The label of elastic fibers inside the granuloma clearly showed that the granuloma territory is not homogeneous (Figs 6, 7).

Our results also showed intragranulomatous expression (in the internal or periovular zone) of substance $\mathrm{P}$ (SP) and vasoactive intestinal peptide (VIP). Substance P, an 11 amino-acid protein, belongs to a class of molecules called tachykinins and is derived from preprotachynin A.
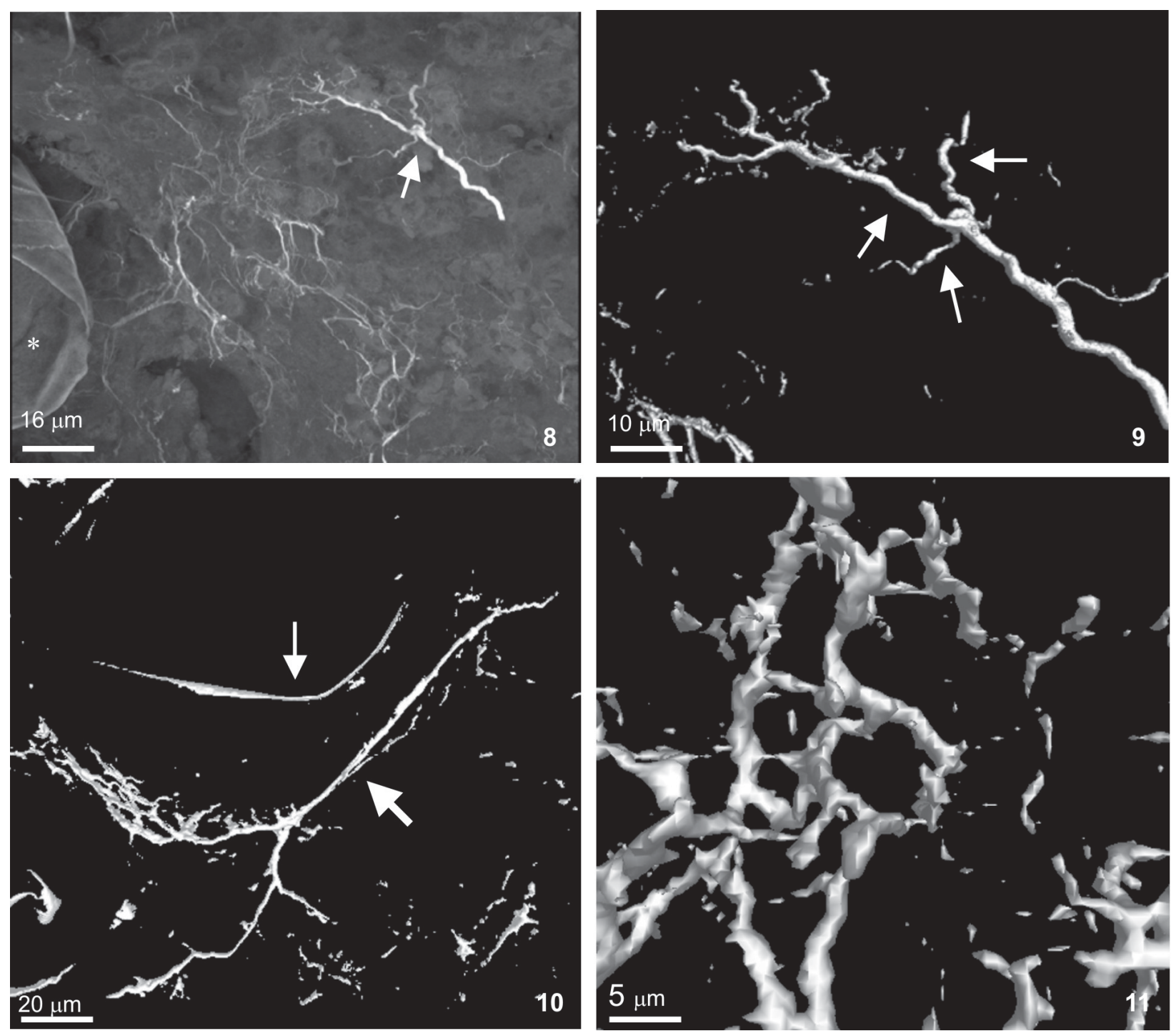

Fig. 8: external or centripetal collagenic growth unit (arrow) distant from the egg (*). Fig. 9: three-dimensional detail of the collagenic growth unit, showing arboriforme branches (arrows). Fig. 10: internal or centrifugal collagenic growth unit (thick arrow), with several branches, located close to the egg (thin arrow). Fig. 11: three-dimensional mesh of collagen fibers made up by collagenic growth units confluence (tomographic confocal images based on PMA-PSR stain). 

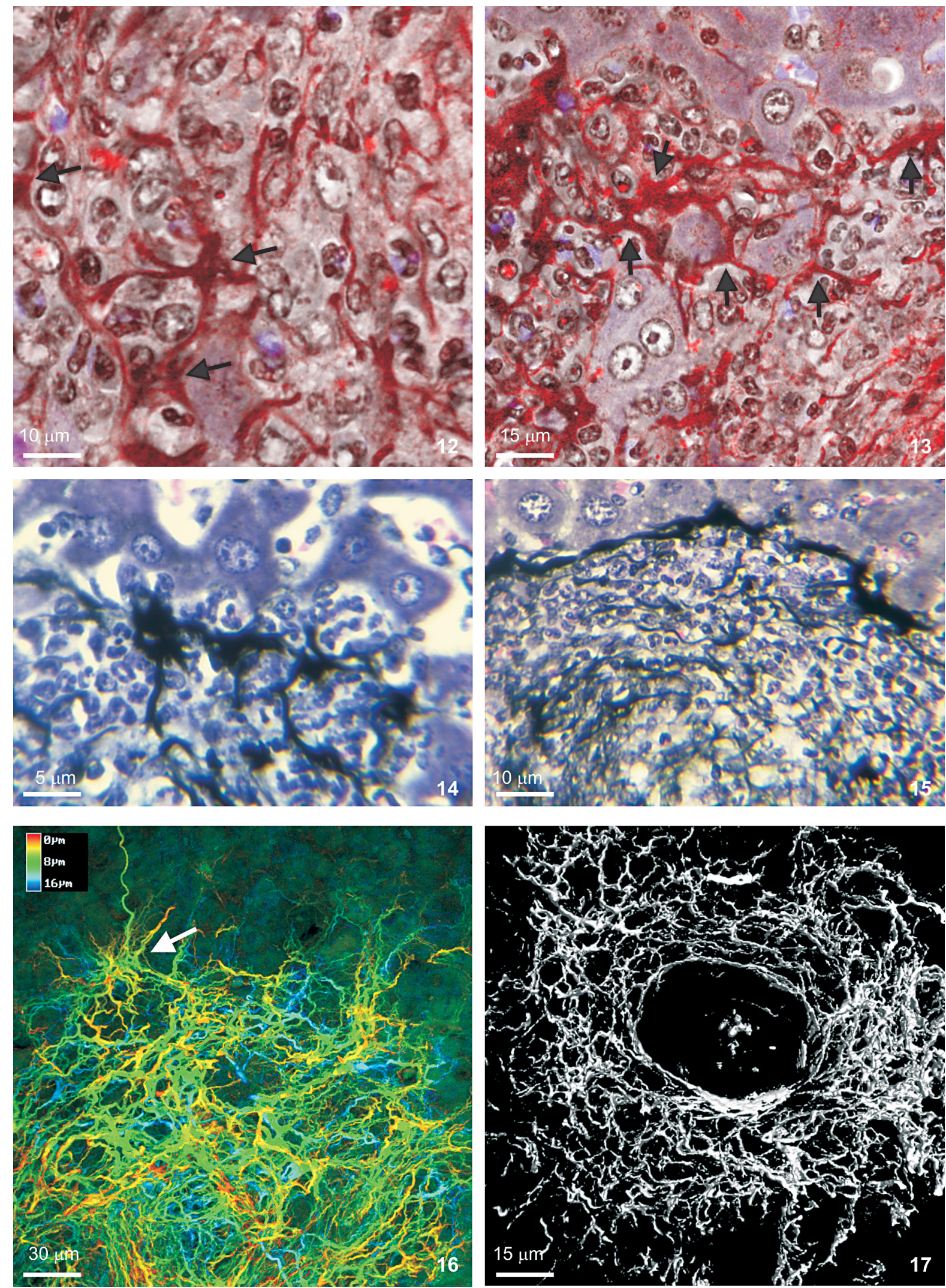

Figs 12, 13: collagenic growth units formed in the interface between three or four cells (Jone's hexamine-silver stain in confocal microscopy). Figs 14, 15: gradual fusion of fiber radiation centers closing externally the granuloma surface (Jone's hexamine-silver stain in bright-field microscopy). Fig. 16: periphery of exudative-productive granuloma showing a clear fiber radiation center (arrow) (Depthcode in confocal microscopy). Fig. 17: final three-dimensional arrangement of interstitial collagens in exudative-productive granuloma (PMA-PS in confocal microscopy). 

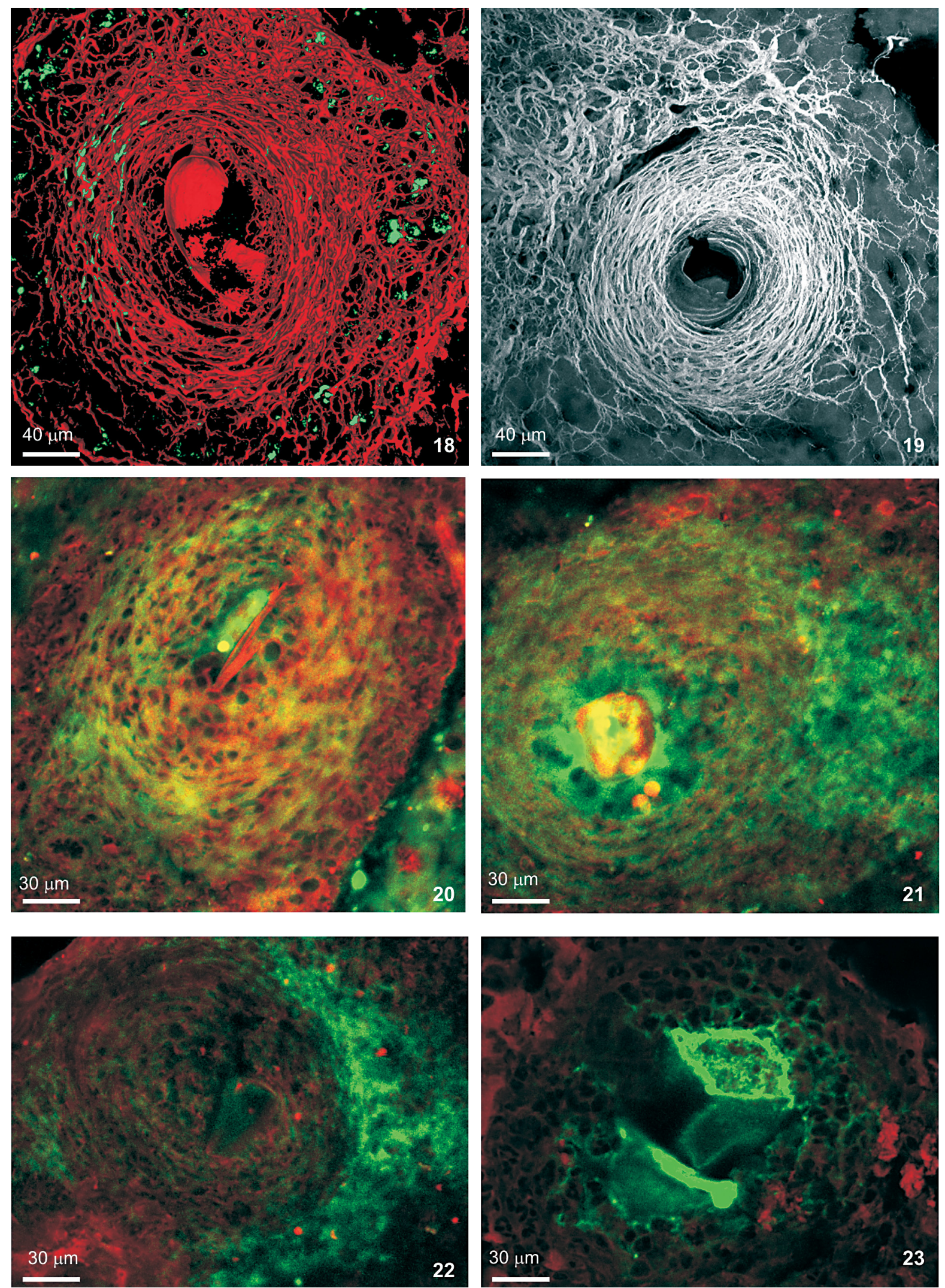

Figs 18, 19: final three-dimensional arrangement of interstitial collagens in exudative-productive (Fig.18) and productive granulomas (Fig. 19). Pigmented macrophages (in green) intermingled with the collagen mesh, mainly in the peripheral zone, are seen in the Fig. 18. In the productive granuloma, the collagen fibers are concentrically arranged (Fig. 19) (PMA-PS in confocal microscopy). Figs 20-23. in situ expression of leptin (Figs 20, 21), receptor of leptin (Ob-R) (Fig. 22) and substance P (Fig. 23) in hepatic schistosome granulomas (confocal indirect immunofluorescence). 
SP interacts with a single high-afinity receptor called neurokinin $1(\mathrm{NK}-1 \mathrm{R})$ on immune cells ( $\mathrm{T}$ cells, macrophages, dendritic cells, and probably other cell types) to enhance IFN-gamma production and amplify the Th1 response (Weinstock 2004, Arsenescu et al. 2005). Here we confirmed in situ production of SP by periovular macrophages. Indeed, Arsenescou et al. (2005) also notice that macrophages are a source for SP at the site of chronic inflammation (schistosome granuloma, spleen and intestinal lamina propria) and IL-12 and STAT4 are regulators of macrophage SP mRNA expression. Vasoactive intestinal peptide (VIP) is also produced by macrophages, which is subject to IL-4 regulation. In schistosome granuloma, T cells express VPAC2 (VIP receptor subtype 2) which is an inducible $\mathrm{CD}^{+} \mathrm{T}$ cell receptor, and its VPAC 2 mRNA is down-modulated in Th2 cells (Metwali et al. 2000). Substance P, but not VIP, also decreases spontaneous immunoglobulin secreting cell number and immunoglobulin secretion in short-term cultures of spleen and granuloma cells (Neil et al. 1991). The granuloma is depleted of innervation and so the neuropeptides, produced mainly by macrophages, can compensate this event, acting like a "granulomatous neural component", taken part in elaborate interactions with the immune and inflammatory cells of the schistosome granuloma as was presented by Sandor et al. (2003). In this sense, VIP and SP modulate immune activity and leptin regulates cytokine balance. The hepatic stellate cells can operate as a fundamental key in this intricate mesh of intrinsic granuloma regulation, because they express a number of neuroendocrine markers (e.g., reelin, nestin, neurotrophins, synaptophysin, and glial-fibrillary acidic protein) and bear receptors for neurotransmitters (Bataller \& Brenner 2005). It is important to point out that hepatic stellate cells are hepatic neuroglia that produce and respond to sympathetic nervous system neurotransmitters to promote hepatic fibrosis (Oben et al. 2004). Leptin is also an endocrine mediator that is partly regulated by other hormones, such as insulin, cortisol, and sex steroids, mainly testosterone. Also, other hormones like thyroid hormone and growth hormone are possibly involved in leptin synthesis (Wauters et al. 2000). A key role in the regulation of leptin production is envisaged for the sympathetic nervous system, operating through b3-adrenoceptors (Trayhurn et al. 1998). It is still unknown what are the functional relationships between leptin and the neuropeptides so far localized inside the schistosome granuloma (Substance P, somatostatin and vasoactive intestinal peptides) (Sandor et al. 2003).

During S. mansoni infection, there is morphological evidence of involvement of various hematopoietic growth factors, which cause eosinophil, monocyte, neutrophil, megakaryocytic, and erythroid extramedullar foci in hepatic granulomas, lymph nodes and omental and mesenteric milky spots (Lenzi et al. 1995, Rossi et al. 1999). In fact, the granuloma, as a complex system, has two types of cellular input: one external (probably from the bone marrow) and other, internal, produced by autopoietic mechanisms. So far, it was impossible to discriminate these two cellular sources that can mobilize more than 40.000 cells/granuloma in the acute phase. The subsequent decrease in the total count of granuloma cells expresses the modulation phenomenon. Moore et al. (1977), in mice lung granulomas induced by intravenous injection of eggs of S. mansoni, which were either unsensitised, sensitized by prior intraperitoneal injection of eggs, or naturally infected with S. mansoni, reported that, in infected mice, the granulomas with the largest cell counts were seen 8 weeks after infection, while the smaller granulomas in the mice infected for 16 or 24 weeks. Here we show that granuloma is rich of cells positive to c-Kit, granulocyte colony stimulating factor receptor, erythropoietin receptor, leptin, and leptin receptor (Ob-R). C-Kit labeled cells in the three zones, mainly in the peripheral one, where the extramedullar hematopoiesis usually occurs, but it also marked stromal cells. The granuloma stroma was shown to produce IL-7 and express c-Kit, and was able to sustain the full B lymphopoiesis in vitro (Rossi et al. 1999). Probably, most of the c-Kit positive cells in the peripheral zone is expressed by eosinophil lineage, in eosinopoiesis foci. Eosinophils have significant levels of surface c-Kit protein, which correspond to stem cell factor receptor (Oliveira et al. 2002). There are in vitro evidences that granulocytemacrophage colony stimulating factor (GM-CSF) is a major mediator in increasing granuloma reaction in human schistosomiasis (Rezende et al. 2004). Although some erythroid foci can be observed in hepatic schistosomal granulomas (Lenzi et al. 1995), the intragranulomatous expression of erythropoietin receptors (Epo-R) appear to be related to other additional or pleiotropic effects of erythropoietin. In its hormonal role, erythropoietin is produced by the kidney in response to oxygen levels and signals the bone marrow to increase the number of circulating erythrocytes (Brines 2002). It has become clear in recent years, however, that erythropoietin and its receptor are members of a cytokine superfamily that mediates diverse functions in nonhematopoietic tissues (Brines 2002). Erythropoietin receptor is widely distributed in the cardiovascular system, including endothelial cells, smooth muscle cells, and cardiomyocytes and preclinical studies have established erythropoietin to be a pleiotropic cytokine with anti-apoptotic activity and tissue-protective actions in the cardiovascular and nervous systems, beyond correction of hemoglobin levels (Manolis et al. 2005). Erythropoietin receptors have been identified also in the central nervous system, retina, vascular endothelium, kidney, lung, liver, gastrointestinal and reproductive tracts and erythroid bone marrow precursors and may present actions other than hematopoiesis stimulation (Lewis 2004). Erythropoietin receptors were also found in human, rat and mouse mesangial, proximal and distal tubular cells; on cells of small bowel of human fetuses and in postnatal enterocytes in rats and humans. This is the first time that Epo-R is reported in hepatic schistosome granuloma and its effect, inside this structure, is still unknown.

Leptin is a JAK2-activating adipokine and both leptin and its receptor share structural and functional similarities with interleukin-6 family of cytokines (Fantuzzi \& Faggioni 2000). Granulomatous leptin is probably produced by activated hepatic stellate cells (HSCs). Leptin colocalizes with a-smooth muscle actin ( $\alpha$-SMA) in activated hepatic stellate cells (Ding et al. 2005) and has sev- 

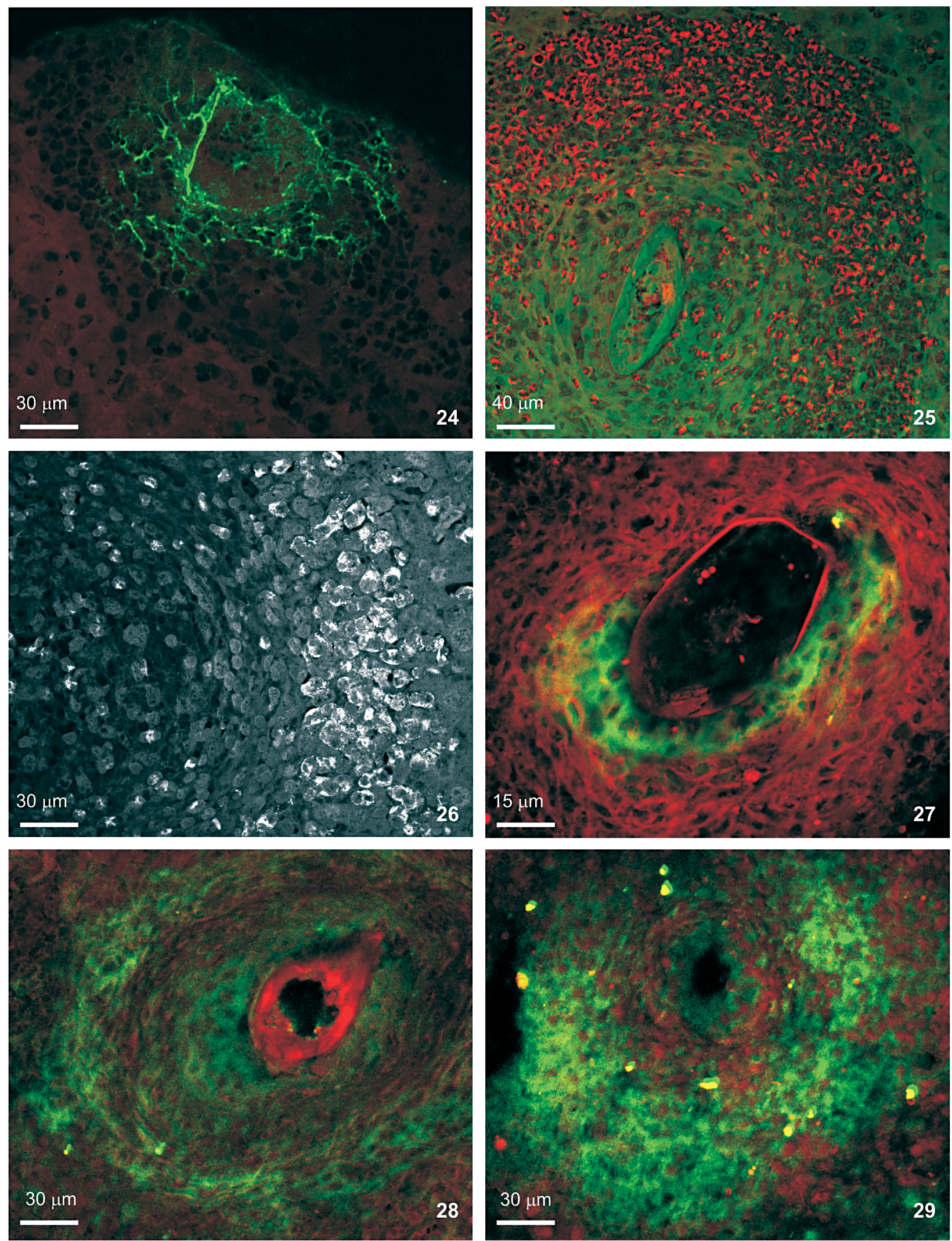

Fig. 24: in situ expression of vasoactive intestinal peptide, predominating in cells close to the egg. Figs 25, 26: eosinopoiesis foci in the peripheral zone. Fig. 27: proliferating macrophages around the egg (Ki-67) (Figs 24 and 27: confocal indirect immunofluorescence: Figs 25 and 26: SR-pH10.2 in confocal microscopy). Fig. 28: Ki-67 proliferating cells mainly in the peripheral and central zones. Fig. 29: in situ expression of c-Kit in hepatic exudative-productive granuloma (confocal indirect immunofluorescence). 
eral fibrogenic activities, expressed by: a) stimulation of tissue inhibitory metalloproteinase-1 (TIMP-1) in activated HSCs; b) enhancement of alfa-2(I) collagen gene expression in HSCs, by stimulation of Stat 3 ; c) increase of Connective Tissue Growth Factor (CTGF) in vitro; d) potent mitogenic activity on HSCs, similar to platelet derived growth factor, and dramatic inhibition of HSCs apoptosis, reducing caspase- 3 activity; e) up-regulation of TGF- $\beta 1$, thereby facilitating tissue repairing and fibrogenesis in sinusoidal microenvironment. Recent studies showed that leptin deficiency decreases but not eliminate hepatic fibrosis produced by S. mansoni (Potter \& Mezey 2002). According to these authors, the effect of leptin in potentiating fibrogenesis is most likely mediated by TGF $\beta 1$.
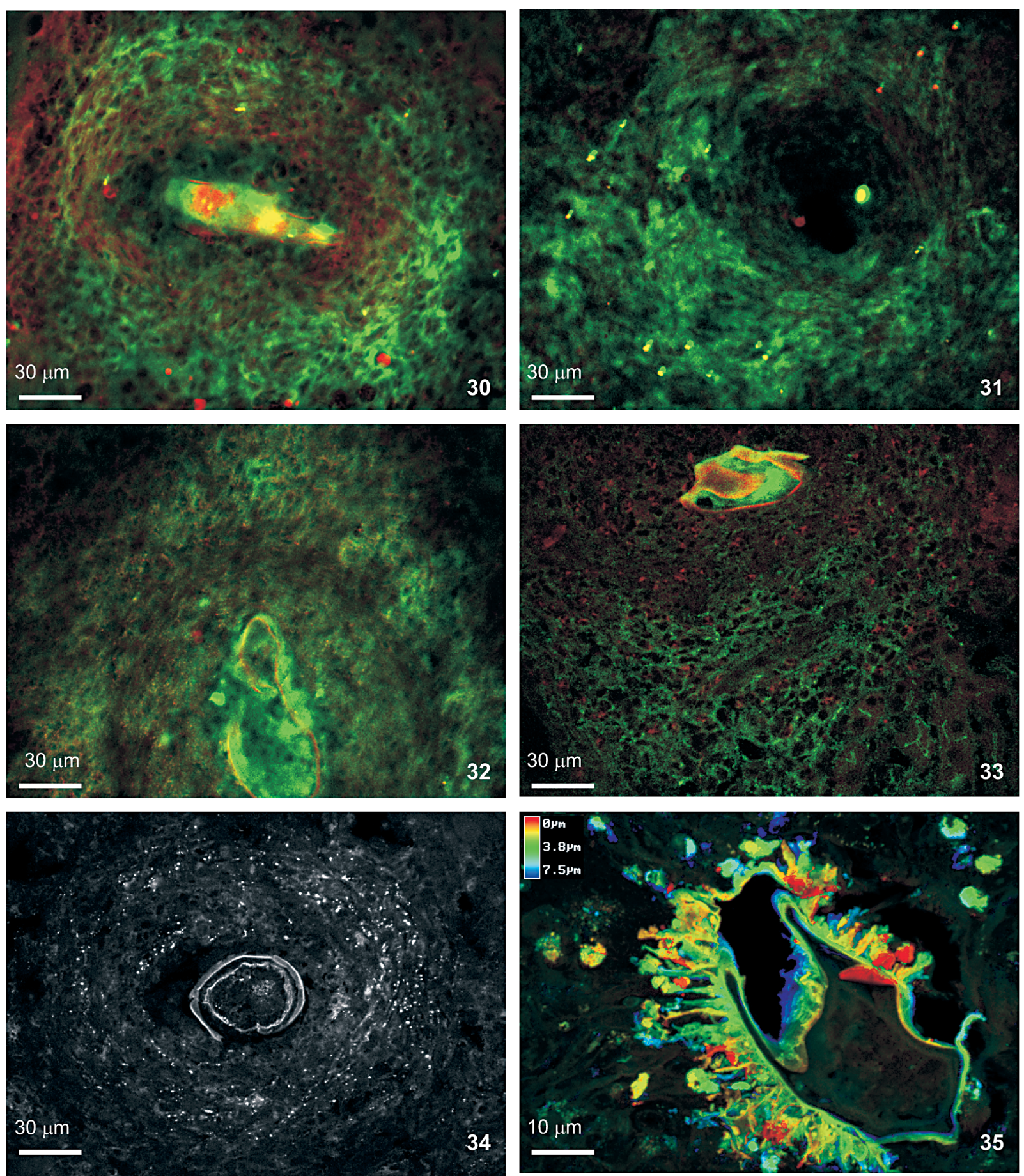

Figs 30-34: in situ expression of granulocyte colony stimulating factor receptor (Fig. 30), erythropoietin receptor (Fig. 31), pan-cadherin (Fig. 32), occludin (Fig. 33), and connexin-43 (Fig. 34) in hepatic schistosome granulomas. Connexin-43 appears as double small dots (confocal indirect immunofluorescence). Fig. 36: crystals of eosinophil proteins on the surface of egg shell, defining Splendore-Hoeppli phenomenon (Depth-code in confocal microscopy). 
$\mathrm{Ob} / \mathrm{ob}$ mice were less able to form granuloma and lung lymphocyte numbers were reduced compared with wildtype mice early during infection with Mycobacterium tuberculosis (Wieland et al. 2005). However, leptin is not only fibrogenic, but is also a pleiotropic hormone, modulating several processes, such as thermogenesis, reproduction, hemostasis, angiogenesis, hematopoiesis, osteogenesis, chondrogenesis, neuroendocrine and immune functions, as well as arterial pressure control, and also affects cytokine production, the activation of monocytes/ macrophages and wound healing (Fantuzzi \& Faggioni 2000, Fietta 2005). In schistosome granuloma, leptin probably act also as a hematopoietic factor. In fact, leptin stimulates the proliferation of murine myelocytic progenitor cells and synergizes with stem cell factor (SCF) in the proliferation of primitive hematopoietic progenitors in vitro.

Another point that we approached in this work was the "granuloma closure", meaning the capacity of the granuloma to limit the diffusion of egg products to adjacent tissues. Here we presented, for the first time in the literature, the in situ presence of pan-cadherin, occluding and connexin- 43 molecules, dramatically strengthening the cellular adhesion and communication among cells of hepatic schistosome granulomas, providing stronger stability. These molecules surely contribute with $\mathrm{Ca}^{2+}$ independent molecules that comprise the immunoglobulin superfamily and integrins, to mediate cell-cell attachment. Integrins are mainly involved in cell-extracellular matrix interactions. Cadherins and integrins establish a link between the internal cytoskeleton of a cell and the exterior of another cell (cadherin) or the extracellular matrix (integrins) (Kierszenbaum 2002). The movements of solutes, ions, and water through an epithelial (granuloma sometimes has an epitheliod arrangement, mainly in the central zone) occurs both across and between individual cell components. The transcellular pathway is controlled by numerous channels and transporters. The paracellular pathway is regulated by a continuous intercellular contact or cell junctions (Kierszenbaum 2002). Occluding junctions (also called tight junctions), composed partially by occluding molecules, have, according to Kierszenbaum (2002), two major functions: (i) they determine epithelial cell polarity by separating the apical domain from the basolateral domain and preventing the free diffusion of lipids and proteins between them; (ii) they prevent the free passage of substances across an epithelial cell layer (paracellular pathway barrier). Then, the paracellular diffusion barrier is created by interactions among occluding, four major proteins (ZO-1, ZO-2, ZO-3, and AF-6) and claudin (Kierszenbaum 2002). Connexins are integral membrane proteins that form communicating junctions, or gap junctions. Connexin-43 was localized in hepatocytes, activated rat hepatic stellate cells, hematopoietic stroma cells, smooth muscle cells, fibroblasts (Ibuki et al. 2002), myofibroblasts (Miragoli et al. 2006), macrophages, thymocytes, and thymic epithelial cells. In our material (data not shown), double stains to connexin-43 combined with eosinophils or thy-1 or MAC-1 or B-220, showed that connexin- 43 was expressed mainly by the middle or fibroblastic-like zone (myofibroblastic cells?) and in lesser amount in the macrophage central zone. In nasal polyps, expression of connexin-43 decreased related to eosinophil expression (Yeh et al. 2005). In exudative-productive granulomas, eosinophils predominated in the peripheral zone, while the connexin- 43 expression was higher in the middle and central zones. The sharing of small metabolites and ions through gap junctions in some tissues provides a mechanism for coordinating the activities of individual cells in such tissues and for smoothing out random fluctuations in small molecules concentrations in different cells. All this mechanism of granuloma closure described above, amplify the barrier effect of other adhesion mol-

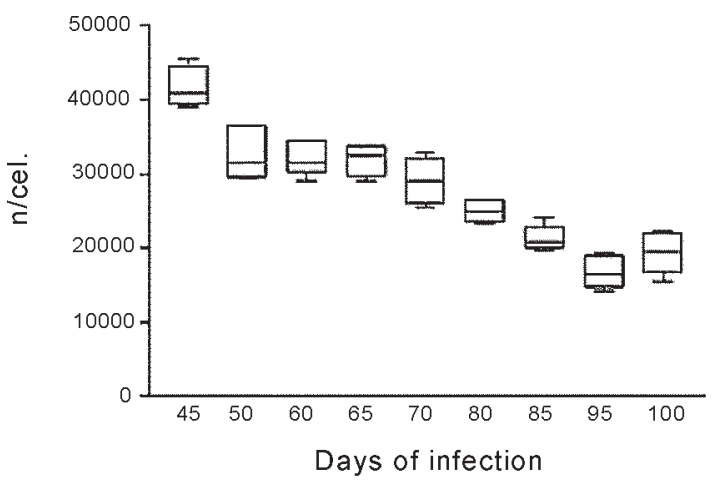

\begin{tabular}{|ccccccccc|}
\hline 45 & 50 & 60 & 65 & 70 & 80 & 85 & 95 & 100 \\
\hline 45500.000 & 36500.00 & 31500.00 & 32500.000 & 30500.00 & 26500.000 & 20125.000 & 14250.000 & 19250.000 \\
41550.000 & 29250.00 & 34500.00 & 30000.000 & 31500.00 & 23250.000 & 21500.000 & 17500.000 & 17500.000 \\
43750.000 & 31500.00 & 34500.00 & 33750.000 & 32750.00 & 25000.000 & 24000.000 & 15250.000 & 22250.000 \\
39000.000 & 36500.00 & 29000.00 & 29000.000 & 27500.00 & 26500.000 & 21250.000 & 19250.000 & 19600.000 \\
39600.000 & 29250.00 & 31500.00 & 32500.000 & 26250.00 & 23250.000 & 20300.000 & 14750.000 & 15500.000 \\
40230.000 & 31500.00 & 31325.00 & 33750.000 & 25500.00 & 25000.000 & 19600.000 & 18500.000 & 21750.000 \\
\hline
\end{tabular}

Fig. 36: total number of cells in isolated hepatic granulomas during different times of schistosomal mansoni infection. The individual counting of isolated granulomas/each liver/time of infection is specified below the graphic. 
ecules described in the schistosome granuloma, such as integrins and molecules from immunoglobulin superfamily (Jacobs \& Van Marck 1998, Jacobs et al. 1998). Periovular degranulated eosinophils, configuring sometimes the Splendore- Hoeppli phenomenon (Fig. 35) and the fusion of fiber irradiation center in the interface between granuloma border and adjacent hepatocytes (Figs 14, 15) also contribute to limit the egg products diffusion, reinforcing the granuloma closure or barrier.

In conclusion, the schistosome granuloma is an extremely complex system with transitory states where the fully organized structure ("little macrophagic metazoa") is literally greater than the sum of actions of its individual cells and extracellular matrix components (Lenzi et al. 1999). The granuloma modulation is not only determined by immune external factors, but may also be influenced by internal constrictions and regulations that need to be better understood.

\section{ACKNOWLEDGMENTS}

To Hallan Souza e Silva and Tiago Rocha Macedo for the excellent contribution to the accurate measurement of fractal dimension.

\section{REFERENCES}

Arsenescu R, Blum AM, Tetwali A, Elliot DE, Weinstock JV 2005. IL-12 induction of mRNA substance $P$ in murine macrophages from the spleen and sites of inflammation. $J$ Immunol 174: 3906-3911.

Bataller R, Brenner DA 2005. Liver fibosis. J Clin Invest 115: 209-218.

Brener Z, Pellegrino J 1956. Method of isolating schistosome granulomas from mouse liver. J Parasitol 42: 564.

Brines M 2002. What evidence supports use of erythropoietin as a novel neurotherapeutic? Oncology (Willinston Park) 16 (Suppl. 10): 79-89.

Carson FL, James MS, Martin JH, Lynn JA 1973. Formalin fixation for electron microscopy: a re-evaluation. Am J Clin Pathol 59: 365-373.

Chong L, Bryan Ray L 2002. Whole-istic biology. Science 295: 1661.

Cross SS 1997. Fractals in pathology. J Pathol 182: 1-8.

Dioguardi N, Franceschini B, Aletti G, Russo C, Grizzi F 2003. Fractal dimension rectified for quantification of liver fibrosis and other irregular microscopic objects. Anal Quant Cytol Histol 25: 312-320.

Dioguardi N, Grizzi F, Bossi P, Roncalli M 1999. Fractal and spectral dimension analysis of liver fibrosis in needle biopsy specimens. Anal Quant Cytol Histol 21: 262-266.

Ding X, Saxena NK, Lin S, Xu A, Srinivasan S, Anania FA 2005. The roles of leptin and adiponectin: a novel paradigm in adipocytokine regulation of liver fibrosis and stellate cell biology. Am J Pathol 166: 1655-1669.

Dolber PC, Spach, MS 1993. Conventional and confocal fluorescence microscopy of collagen fibers in the heart. $J$ Histochem Cytochem 41: 465-469.

Fantuzzi G, Faggioni R 2000. Leptin in the regulation of immunity, inflammation, and hematopoiesis. J Leukoc Biol 68: 437-446.
Fietta P 2005. Focus on leptin, a pleiotropic hormone. Minerva Med 96: 65-75.

Ibuki N, Yamaoka Y, Sawa Y, Kawasaki T, Yoshida S 2002. Different expressions of connexin 43 and 32 in the fibroblasts of human dental pulp. Tissue Cell 34: 170-176.

Jacobs W, Van Marck E 1998. Adhesion and co-stimulatory molecules in the pathogenesis of hepatic and intestinal schistosomiasis mansoni. Mem Inst Oswaldo Cruz 93: 523-529.

Jacobs W, Bogers JJ, Timmermans JP, Deelder AM, Van Marck EA 1998. Adhesion molecules in intestinal Schistosoma mansoni infection. Parasitol Res 84: 276-280.

Kierszenbaum AL 2002. Histology and Cell Biology: an Introduction to Pathology, Mosby, St. Louis, Missouri, 619 pp.

Landini G 1996. Applications of fractal geometry in pathology. In PM Iannaccone, M Khokha (eds), Fractal Geometry in Biological Systems: an Analytical Approach, CRC Press, New York, p. 205-246.

Lenzi HL, Romanha WS 2003. Schistosomal granuloma is like a small multicellular organism that operates as a complex system. In R Mondaini, Proceedings of the Second brazilian Symposium on Mathematical and Computational Biology, e-papers, Rio de Janeiro, p. 257-282.

Lenzi HL, Kimmel E, Schechtman H, Pelajo-Machado M, Romanha WS, Pacheco RG, Mariano M, Lenzi JA 1998. Histoarchitecture of schistosomal granuloma development and involution: morphogenetic and biomechanical approaches. Mem Inst Oswaldo Cruz 93 (Suppl. I): 141-151.

Lenzi HL, Kimmel E, Schechtman H, Pelajo-Machado M, Vale BS, Panasco MS, Lenzi JA 1999. Collagen arrangement in hepatic granuloma in mice infected with Schistosoma mansoni: dependence on fiber radiation centers. Braz J Med Biol Res 32: 639-643.

Lenzi HL, Lenzi JA, Rosman FC, Pelajo-Machado M, Mota EM, Panasco MS, Oliveira DN 1995. Extramedullary hematopoiesis in murine schistosomiasis mansoni. Mem Inst Oswaldo Cruz 90: 169-177.

Lenzi HL, Romanha WS, Pelajo-Machado M 2004. Possíveis níveis de complexidade na modelagem de sistemas biológicos - Pode a matemática coreografar a dança biológica? In EXL de Andrade, R Sampaio, GN Silva (eds), Notas em Matemática Aplicada: Modelagem em Biomatemática. Sociedade Brasileira de Matemática Aplicada e Computacional, São Carlos, SP, p. 54-78.

Lewis LD 2004. Preclinical and clinical studies: a preview of potential future applications of erythropoietic agents. Semin Hematol 41(Suppl.7): 17-25.

Manolis AS, Tzeis S, Triantafyllou K, Michaelidis J, Pyrros I, Sakellaris N, Kranidis A, Melita H 2005. Erythropoietin in heart failure and other cardiovascular diseases: hematopoietic and pleiotropic effects. Curr Drug Targets Cardiovasc Haematol Disord 5: 355-375.

Metwali A, Blum AM, Li J, Elliott DE, Weinstock JV 2000. IL4 regulates VIP receptor subtype 2 mRNA (VPAC2) expression in T cells in murine schistosomiasis. FASEB $J 14$ : 948-954.

Miragoli M, Gaudesius G, Rohr S 2006. Eletrotonic modulation of cardiac impulse conduction by myofibroblasts. Circ Res [Epub ahead of print] 
Moal F, Chappard D, Wang J, Vuillemin E, Michalak-provost S, Rousselet MC, Oberti F, Cales P 2003. Fractal dimension can distinguish models and pharmacologic changes in liver fibrosis in rats. Hepatology 37: 483-484.

Moore DL, Grove DI, Warren KS 1977. The Schistosomal mansoni egg granuloma: quantitation of cell population. J Pathol 121: 41-50.

Neil GA, Blum A, Weinstock JV 1991. Substance P but not vasoactive intestinal peptide modulates immunoglobulin secretion in murine schistosomiasis. Cell Immunol 135: 394-401.

Oben JA, Roskams T, Yang S, Lin H, Sinelli N, Torbenson M, Smedh U, Moran TH 2004. Hepatic fibrogenesis requires sympathetic neurotransmitters. Gut 53: 438-445.

Oliveira SH, Taub DD, Nagel J, Smith R, Hogaboam CM, Berlin A, Lukacs NW 2002. Stem cell factor induces eosinophil activation and degranulation: mediator release and gene array analysis. Blood 100: 4291-4297.

Potter JJ, Mezey E 2002. Leptin deficiency reduces but does not eliminate the development of hepatic fibrosis in mice infected with Schistosoma mansoni. Liver 22: 173-177.

Rezende CM, Goes TS, Goes VS, Avedo V, Leit MF, Goes AM 2004. GM-CSF and TNF-alpha synergize to increase in vitro granuloma size of PBMC from humans induced by Schistosoma mansoni recombinant $28-\mathrm{kDa}$ GST. Immunol
Lett 95: 221-228.

Rossi MI, Dutra HS, El-Cheikh MC, Bonomo A, Borojevic R 1999. Extramedullar B lymphopoiesis in liver schistosomal granulomas: presence of the early stages and inhibition of the full B cell differentiation. Int Immunol 11: 509-518.

Sandor M, Weinstock JV, Wynn TA 2003. Granulomas in schistosome and mycobacterial infections: a model of local immune responses. Trends Immunol 24: 44-52.

Trayhurn P, Hoggard N, Mercer JG, Rayner DV 1998. Hormonal and neuroendocrine regulation of energy balance the role of leptin. Arch Tierernahr 51: 177-185.

Wauters M, Considine RV, Van Gaal LF 2000. Human leptin: from an adipocyte hormone to an endocrine mediator. Eur $J$ Endocrinol 143: 293-311.

Weinstock JV 2004. The role of substance P, hemokinin and their receptor in governing mucosal inflammation and granulomatous responses. Front Biosci 9: 1936-1943.

Wieland CW, Florquin S, Chan ED, Leeman JC, Weijer S, Verbon A, Fantuzzi G, van der Poll T 2005. Pulmonary Mycobacterium tuberculosis infection in leptin-deficient ob/ob mice. Int Immunol 17: 1399-1408.

Yeh TH, Hsu WC, Chen YS, Hsu CJ, Lee SY 2005. Decreased connexin 43 expression correlated with eosinophil infiltration in nasal polyps. Am J Rhinol 19: 59-64. 